\title{
Effect of purified omega-3 fatty acids on reducing left ventricular remodeling after acute myocardial infarction (OMEGA-REMODEL study): a double-blind randomized clinical trial)
}

\author{
Bobby Heydari ${ }^{2,1^{*}}$, Siddique A Abbasi ${ }^{2}$, Ravi Shah ${ }^{2}$, Shuaib Abdullah ${ }^{6}$, Jiazuo Feng ${ }^{2}$, William Harris ${ }^{5}$, Joe McConnell', \\ Evan Appelbaum ${ }^{4}$, Udo Hoffmann ${ }^{3}$, Michael Steigner ${ }^{2}$, Ron Blankstein², Elliott A Antman², Michael Jerosch-Herold², \\ Raymond Y Kwong ${ }^{2}$
}

From 18th Annual SCMR Scientific Sessions

Nice, France. 4-7 February 2015

\section{Background}

Acute myocardial infarction (MI) remains a leading cause of patient death and morbidity. Prognosis following MI has been shown to be strongly associated with small changes in left ventricular remodeling. Omega-3 fatty acid (PUFAs) supplementation may have a number of beneficial pleiotropic effects, including enhancement of myocardial relaxation ${ }^{1}$ and reduction of vascular tone. ${ }^{2}$ One prospective, randomized trial demonstrated significant survival benefit for PUFAs following acute MI. ${ }^{3}$ However, the mechanism and potential myocardial changes during the convalescent phase post MI from PUFAs have not been well described.

\section{Methods}

The OMEGA-REMODEL study was a randomized, double-blinded, placebo controlled trial of PUFA supplementation post acute MI. A total of 358 patients were randomized to study therapy with PUFAs $(n=180)$ or matching placebo $(n=178)$ and underwent baseline assessment by CMR 4-28 days following MI, with followup after 6 months of randomized therapy. The primary endpoint was changes in left ventricular end-systolic volume indexed to body surface area (LVESVI). Secondary outcomes included a) change in total infarct size, b) expansion of MECVF within noninfarcted myocardium, and c) changes in systemic biomarkers.

${ }^{2}$ Cardiology, Brigham and Women's Hospital, Boston, MA, USA Full list of author information is available at the end of the article

\section{Results}

Baseline demographics for the entire cohort and both treatment arms are shown in Table 1. CMR characteristics, PUFAs levels, and biomarkers for baseline and 6-month post-treatment values of each treatment arm are shown in Table 2 and Figure 1. There were no significant differences for any ventricular parameters, infarct size or MECVF at baseline. After 6 months of therapy, follow-up CMR revealed a significant difference for percent change of LVESVI (-5.4 \pm 16.6 PUFAs versus $0.75 \pm 21.0$ Placebo, $\mathrm{p}=0.01)$ and MECVF $(-1.3 \pm 14.9$ PUFAs versus $3.8 \pm 18.0$ Placebo, $\mathrm{p}=<0.05)$ between the treatment arms. In addition, the inflammatory biomarkers, hsCRP and myeloperoxidase, were substantially reduced in the PUFAs treated arm as compared with the placebo arm $(-98.2 \pm 243.6 \%$ versus $-30.5 \pm 221.5 \%$ $\mathrm{p}<0.01$, and $-0.8 \pm 6.7$ versus $+0.8 \pm 5.3 \mathrm{p}=<0.05$, respectively). Finally, the percent reduction in ST2 after 6 months, a biomarker of myocardial fibrosis, was significantly greater in the PUFAs treated arm $(-1.35 \pm 8.0$ versus $+0.92 \pm 7.5, \mathrm{p}=0.03)$. Adjusted multivariable analysis demonstrated an independent association for change in PUFAs levels with adverse LV remodeling.

\section{Conclusions}

The results of this study demonstrated a significant reduction in LVESVI and MECVF for patients treated with high-dose PUFAs as compared to placebo. Further assessment of systemic biomarkers demonstrated a substantial reduction in biomarkers of inflammation, and 
Table 1. Baseline Demographics

\begin{tabular}{|c|c|c|c|c|}
\hline Characteristics & $\begin{array}{c}\text { All patients } \\
(\mathrm{n}=358)\end{array}$ & $\begin{array}{c}\text { Treatment PUFAs } \\
\qquad(n=180)\end{array}$ & $\begin{array}{l}\text { Placebo } \\
(\mathrm{n}=178)\end{array}$ & P value \\
\hline Age (years) & $58.9 \pm 10.5$ & $59.6 \pm 10.4$ & $58.3 \pm 10.4$ & 0.25 \\
\hline Female, $n(\%)$ & $69(19)$ & $31(17)$ & $38(21)$ & 0.42 \\
\hline Body surface area, $\mathrm{m}^{2}$ & $1.99 \pm 0.2$ & $1.99 \pm 0.2$ & $2.0 \pm 0.2$ & 0.71 \\
\hline Heart rate (beats/min) & $66.1 \pm 12.0$ & $65.3 \pm 10.0$ & $67.0 \pm 13.4$ & 0.19 \\
\hline Systolic blood pressure $(\mathrm{mm} \mathrm{Hg})$ & $121 \pm 16$ & $121 \pm 15$ & $120 \pm 16$ & 0.75 \\
\hline Diastolic blood pressure $(\mathrm{mm} \mathrm{Hg})$ & $70 \pm 10$ & $70 \pm 10$ & $70 \pm 11$ & 0.62 \\
\hline $\begin{array}{l}\text { Ethnicity } \\
\quad \text { White, } n(\%)\end{array}$ & $289(81)$ & $143(79)$ & $146(82)$ & 0.78 \\
\hline $\begin{array}{l}\text { CAD History } \\
\text { CHF, } n(\%) \\
\text { MI, } n(\%) \\
\text { PCI, } n(\%) \\
\text { CABG, } n(\%)\end{array}$ & $\begin{array}{l}10(3) \\
36(10) \\
40(11) \\
35(10)\end{array}$ & $\begin{array}{c}4(2) \\
22(12) \\
21(12) \\
24(14)\end{array}$ & $\begin{array}{c}6(3) \\
14(8) \\
19(11) \\
11(6)\end{array}$ & $\begin{array}{l}0.75 \\
0.99 \\
0.73 \\
<0.05 \\
\end{array}$ \\
\hline $\begin{array}{l}\text { Cardiac risk factors, } n(\%) \\
\text { Hypercholesterolemia } \\
\text { Diabetes mellitus } \\
\text { Hypertension } \\
\text { Smoking }\end{array}$ & $\begin{array}{l}253(71) \\
90(25) \\
229(64) \\
177(49)\end{array}$ & $\begin{array}{c}133(74) \\
45(25) \\
117(65) \\
85(47)\end{array}$ & $\begin{array}{l}120(67) \\
45(25) \\
112(63) \\
92(52)\end{array}$ & $\begin{array}{l}0.13 \\
0.99 \\
0.58 \\
0.52\end{array}$ \\
\hline $\begin{array}{l}\text { Medications, } n(\%) \\
\text { Aspirin } \\
\beta \text {-adrenergic blockers } \\
\text { Statin } \\
\text { Calcium channel blockers } \\
\text { ACE inhibitor/ARB } \\
\text { Insulin } \\
\end{array}$ & $\begin{array}{c}356(99) \\
326(91) \\
342(96) \\
26(7) \\
260(73) \\
33(9) \\
\end{array}$ & $\begin{array}{c}179(99) \\
162(90) \\
171(95) \\
17(9) \\
133(74) \\
18(10) \\
\end{array}$ & $\begin{array}{l}177(99) \\
162(92) \\
171(96) \\
9(5) \\
127(71) \\
15(8) \\
\end{array}$ & $\begin{array}{l}0.99 \\
0.85 \\
0.99 \\
0.23 \\
0.47 \\
0.59 \\
\end{array}$ \\
\hline $\begin{array}{l}\text { Type MI } \\
\text { STEMI } \\
\text { Anterior }\end{array}$ & $\begin{array}{l}203(57) \\
96(27)\end{array}$ & $\begin{array}{l}100(56) \\
48(27)\end{array}$ & $\begin{array}{l}103(58) \\
48(27)\end{array}$ & $\begin{array}{l}0.83 \\
0.99 \\
\end{array}$ \\
\hline TIMI 3 Flow Achieved & $327(91)$ & $164(92)$ & $163(92)$ & 0.99 \\
\hline $\begin{array}{l}\text { Laboratory } \\
\text { Hematocrit, } \% \\
\text { AST, } \mu \mathrm{mol} / \mathrm{L} \\
\text { Creatinine, } \mu \mathrm{mol} / \mathrm{L} \\
\text { Glucose, } \mathrm{mg} / \mathrm{dl} \\
\text { Peak troponin }(\mathrm{T}), \mu \mathrm{mol} / \mathrm{L} \\
\text { Peak creatine kinase, U/L } \\
\text { Peak creatine kinase MB, U/L }\end{array}$ & $\begin{array}{c}39.0 \pm 26.4 \\
42.7 \pm 78.2 \\
0.93 \pm 0.24 \\
124.9 \pm 63.4 \\
20 \pm 62 \\
1166 \pm 1269 \\
98 \pm 97\end{array}$ & $\begin{array}{c}39.4 \pm 36.8 \\
48.3 \pm 76.0 \\
0.95 \pm 0.26 \\
124.6 \pm 67.3 \\
26 \pm 81 \\
1139 \pm 1067 \\
98 \pm 95\end{array}$ & $\begin{array}{c}38.7 \pm 7.9 \\
37.3 \pm 80.1 \\
0.91 \pm 0.22 \\
125.1 \pm 59.7 \\
15 \pm 34 \\
1193 \pm 1450 \\
97 \pm 100\end{array}$ & $\begin{array}{l}0.79 \\
0.20 \\
0.14 \\
0.90 \\
0.12 \\
0.72 \\
0.98 \\
\end{array}$ \\
\hline
\end{tabular}

Data listed where available. Abbreviations: $\mathrm{ACE}=$ angiotensin converting enzyme, $\mathrm{ARB}=$ angiotensinx receptor blocker, $\mathrm{AST}=$ aspartate aminotransferase, $\mathrm{CAD}=$ coronary artery disease, $\mathrm{CABG}=$ coronary artery bypass grafting, $\mathrm{CHF}=$ congestive heart failure, $\mathrm{MI}=$ myocardial infarction, $\mathrm{PCI}=$ percutaneous coronary intervention, PUFA = purified omega-3 fatty acids, STEMI = ST elevation myocardial infarction, TIMI = 'thrombolysis in myocardial infarction'

Figure 1 Baseline demographics

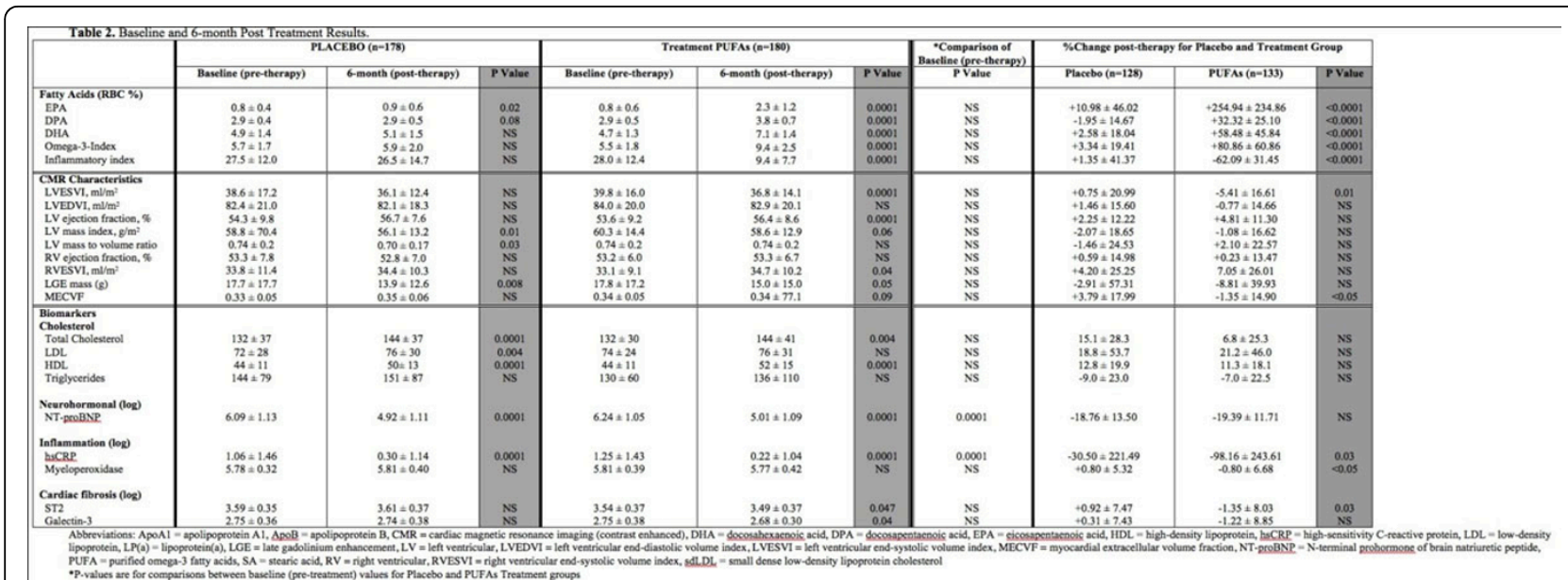

Figure 2 Baseline and 6-month post treatment results 
fibrosis for the PUFAs treated arm. These findings represent the first description of the effect of PUFAs on myocardial tissue phenotypes during the convalescent phase post MI and may suggest potentially important pathophysiological pathways for their pleiotropic effects.

\section{Funding}

National Institutes of Health Heart, Lung and Blood Institute (NHLBI).

\section{Authors' details}

${ }^{1}$ Cardiology, University of Calgary, Calgary, AB, Canada. ${ }^{2}$ Cardiology, Brigham and Women's Hospital, Boston, MA, USA. ${ }^{3}$ Radiology, Massachusetts General Hospital, Boston, MA, USA. ${ }^{4}$ Cardiology, BIDMC, Boston, MA, USA. ${ }^{5}$ Health Diagnostic Laboratory, Richmond, VA, USA. ${ }^{6}$ Cardiology, UT Southwestern, Dallas, TX, USA.

Published: 3 February 2015

doi:10.1186/1532-429X-17-S1-O7

Cite this article as: Heydari et al:. Effect of purified omega- 3 fatty acids on reducing left ventricular remodeling after acute myocardial infarction (OMEGA-REMODEL study): a double-blind randomized clinical trial). Journal of Cardiovascular Magnetic Resonance 2015 17(Suppl 1):O7.

Submit your next manuscript to BioMed Central and take full advantage of:

- Convenient online submission

- Thorough peer review

- No space constraints or color figure charges

- Immediate publication on acceptance

- Inclusion in PubMed, CAS, Scopus and Google Scholar

- Research which is freely available for redistribution

Submit your manuscript at www.biomedcentral.com/submit
C Biomed Central 